\title{
Single-Charge Sensitivity of Single-Walled Carbon Nanotube Multifunctional Quantum Transistor
}

\author{
Takafumi Kamimura ${ }^{1,2,3,{ }^{*}}$ and Kazuhiko Matsumoto ${ }^{1,3,4}$ \\ ${ }^{1}$ Advanced Industrial Science and Technology (AIST), \\ 1-1-1 Umezono Tsukuba, Ibaraki 305-8568, Japan \\ ${ }^{2}$ Japan Society for the Promotion of Science (JSPS), \\ 6 Ichibancho, Chiyoda-ku, Tokyo 102-8471, Japan \\ ${ }^{3}$ CREST, Japan Science and Technology Agency (JST), Kawaguchi Center Building 4-1-8, \\ Honcho, Kawaguchi-shi, Saitama 332-0012, Japan \\ ${ }^{4}$ The Institute of Scientific and Industrial Research, Osaka University, \\ Mihogaoka 8-1, Ibaraki, Osaka 567-0047, Japan
}

(Received February 25, 2009; accepted May 12, 2009)

Key words: $\quad$ single-walled carbon nanotube, resonant tunneling transistor, single-hole transistor, single-charge sensitivity

Single-walled carbon nanotube (SWNT) field-effect transistors show hysteresis in their electrical characteristics owing to the amorphous carbon around the carbon nanotube. Here, we show the reduction in the hysteresis characteristic by a refining process applied repeatedly to the carbon nanotube. Moreover, an SWNT multifunctional quantum transistor that shows a transition between resonant transistor (RTT) characteristics and single-hole transistor (SHT) characteristics with a change in applied gate voltage is developed. By fabricating a few charge storage units around the channel of a purified SWNT multifunctional quantum transistor, an abrupt discrete switching of the source-drain current is observed, which corresponds to the single-charge transition between a storage unit and an SWNT. In addition, storage energy was controlled by adjusting applied bias.

\section{Introduction}

Various electrode materials for single-walled carbon nanotube (SWNT) transistors were investigated. Pd electrodes have been used for ohmic contacts, ${ }^{(1)} \mathrm{Ti}$ electrodes for Schottky contacts for hole conduction, ${ }^{(2-4)}$ and $\mathrm{Mg}$ and $\mathrm{Ca}$ electrodes for electron conduction. ${ }^{(5)}$ For sub-micron-order channel lengths at low temperatures, SWNT transistors with ohmic contacts have shown resonant tunneling transistor (RTT) characteristics, ${ }^{(6)}$ which are also called Fabry-Perot characteristics, and SWNT transistors with Schottky contacts have shown single-hole transistor (SHT) characteristics, ${ }^{(7)}$ in

*Corresponding author: e-mail: t.kamimura@aist.go.jp 
which Schottky barriers act as tunneling barriers. Therefore, electrode materials should be carefully chosen to obtain the desired characteristics.

In this study, we succeeded in fabricating a multifunctional quantum transistor using the particle and wave natures of holes in an SWNT. This transistor can operate as an RTT and also as an SHT. An RTT is a device that uses the wave nature of holes and an SHT uses the particle nature of holes in the SWNT. Both devices need tunneling barriers on both sides of the quantum island. The RTT needs a strong coupling while the SHT needs a weak coupling between the quantum island and the electrodes. Usually, these tunneling barriers are fabricated from thin oxide layers. Therefore, the thickness of the tunneling barriers and coupling strength cannot normally be controlled in a given device. In the present device, however, the Schottky barriers act as tunneling barriers between the SWNT quantum island and the electrodes. Therefore, the thickness of the tunneling barriers and the coupling strength between the SWNT and the electrodes can be controlled by regulating the applied gate voltage $V_{\mathrm{G}}$.

Moreover, an SWNT is a cylinder with a diameter of several nanometers. The small diameter makes it possible to detect an electrical field from even a single charge. Moreover, by observing the relative energy difference between the conducting carrier and the single charges to be measured, it is possible to define the potential energy of the single charges to be measured. However, as described in many reports, ${ }^{(8,9)}$ SWNT electron devices show hysteresis characteristics in gate voltage-drain current characteristics. The hysteresis characteristics are caused by a gate-voltage-dependent charge fluctuation, e.g., adsorption of water molecules around an SWNT, ${ }^{(8)}$ charging into an insulator layer around an SWNT, ${ }^{(9)}$ and charging into amorphous carbon around an SWNT.(3) By eliminating these origins of the hysteresis characteristics, the number of fluctuating charges decreases and a single charge can be detected by an SWNT multifunctional quantum transistor.

\section{Materials and Methods}

\subsection{Method}

We have eliminated the three main origins of the hysteresis characteristics of an SWNT field-effect transistor identified in recent reports. ${ }^{(3,8-10)}$ To burn amorphous carbon, we annealed an SWNT at low temperature in an oxidizable atmosphere. ${ }^{(10)}$ To reduce the number of adsorbed atmospheric molecules, we covered the channel with a silicon dioxide layer. To reduce the number of trap sites in the insulator, we reduced the channel length to $73 \mathrm{~nm}$. The SWNT multifunctional quantum transistor fabricated by the process mentioned above shows almost no hysteresis characteristics in the gate voltage range from -40 to $40 \mathrm{~V}$. Moreover, an abrupt discrete switching of the sourcedrain current is observed in the electrical measurements of the SWNT multifunctional quantum transistor at $7.3 \mathrm{~K}$. These random telegraph signals (RTSs) are attributed to charge-fluctuating charge traps near the SWNT multifunctional quantum transistor conduction channel. The current-switching behavior associated with the occupation of individual electron traps is demonstrated and analyzed statistically. 


\subsection{Sample preparations}

A schematic of the sample structure is shown in Fig. 1. The SWNT was prepared as follows. An $\mathrm{n}^{+}$-Si wafer with a thermally grown 300 -nm-thick oxide was used as a substrate. Layered $\mathrm{Fe} / \mathrm{Mo} / \mathrm{Si}(2 \mathrm{~nm} / 20 \mathrm{~nm} / 40 \mathrm{~nm})$ catalysts were evaporated using an electron-beam evaporator under a vacuum of $10^{-6} \mathrm{~Pa}$. These layered catalysts were patterned on the substrate using conventional photolithography. The SWNT was grown by thermal chemical vapor deposition (CVD) using mixed gases of hydrogen and argonsparged ethanol. After the growth of the SWNT, it was purified by burning amorphous carbon around the SWNT in an air atmosphere at a temperature of several hundred degrees Celsius. ${ }^{(10)} \mathrm{Ti}(30 \mathrm{~nm})$ electrodes were deposited on the patterned catalysts as the source and drain, and on the back surface of the $\mathrm{n}^{+}$-Si substrate for the gate, using an electron-beam evaporator under a vacuum of $10^{-6} \mathrm{~Pa}$. The distance $(L)$ between the source and drain was $73 \mathrm{~nm}$. Thus, a back-gate-type multifunctional quantum transistor with an SWNT channel that had the functions of an RTT and an SHT was fabricated. A single-charge measurement was carried out with a structure in which a silicon dioxide layer is on the SWNT channel.

\section{Results and Discussions}

Figure 2(a) shows the differential conductance $\mathrm{d} I_{\mathrm{D}} / \mathrm{d} V_{\mathrm{D}}$ characteristic as a function of $V_{\mathrm{G}}$ at $7.3 \mathrm{~K}$, where drain voltage was set at $8 \mathrm{mV}$. An oscillation characteristic with two oscillation periods is also observed in Fig. 2(a). A large oscillation period of $\Delta V_{\mathrm{G}}=1.1 \mathrm{~V}$ was observed at $V_{\mathrm{G}} \geq-16 \mathrm{~V}$ and a small oscillation period of $\Delta V_{\mathrm{G}}$ $=0.65 \mathrm{~V}$ was observed at $V_{\mathrm{G}} \leq-16 \mathrm{~V}$. Figure $2(\mathrm{~b})$ shows the $\mathrm{d} I_{\mathrm{D}} / \mathrm{d} V_{\mathrm{D}}$ peak on a linear scale at $V_{\mathrm{G}} \geq-16 \mathrm{~V}$ and $V_{\mathrm{G}} \leq-16 \mathrm{~V}$. A clear difference in oscillation period is observed as follows. The peaks of $\mathrm{d} I_{\mathrm{D}} / \mathrm{d} V_{\mathrm{D}}$ at $V_{\mathrm{G}} \geq-16 \mathrm{~V}$ are well fitted by a Gaussian, i.e., $G \approx \frac{2 e^{2}}{h} \frac{T_{L} T_{R}}{\Gamma} \frac{1}{2 \mathrm{k} T} \exp \left(\frac{\varepsilon_{\mathrm{F}}-\varepsilon_{0}}{2 \mathrm{k} T}\right)$, and at $V_{\mathrm{G}} \leq-16 \mathrm{~V}$ by a Lorentzian, i.e.,

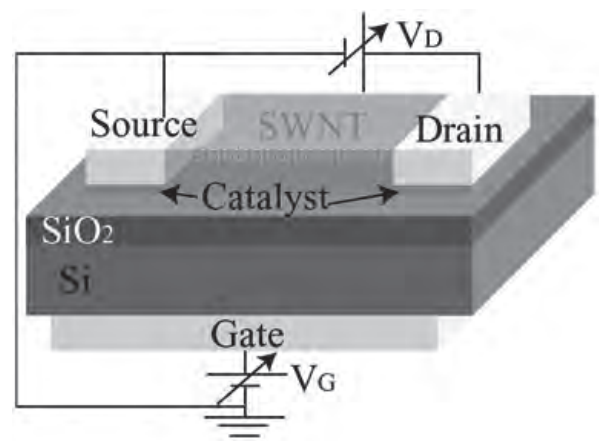

(a)

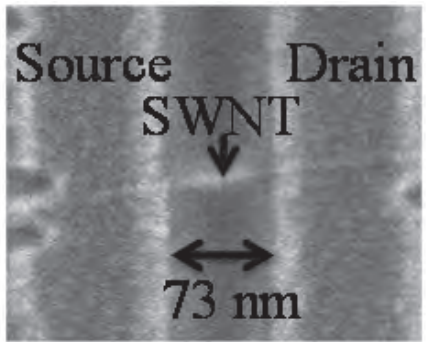

(b)

Fig. 1. Schematic structure of SWNT multifunctional quantum transistor covered by silicon dioxide layer. The channel length is $73 \mathrm{~nm}$. The inset shows a SEM image around the channel before silicon dioxide deposition. A few charge storages are fabricated in the $\mathrm{SiO}_{2}$ layer. 


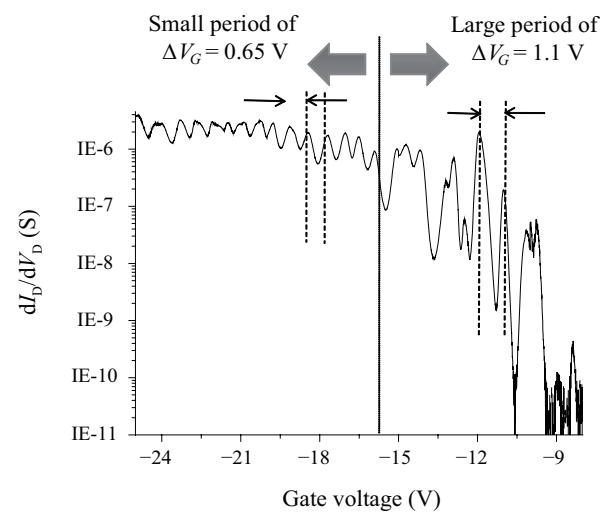

(a)
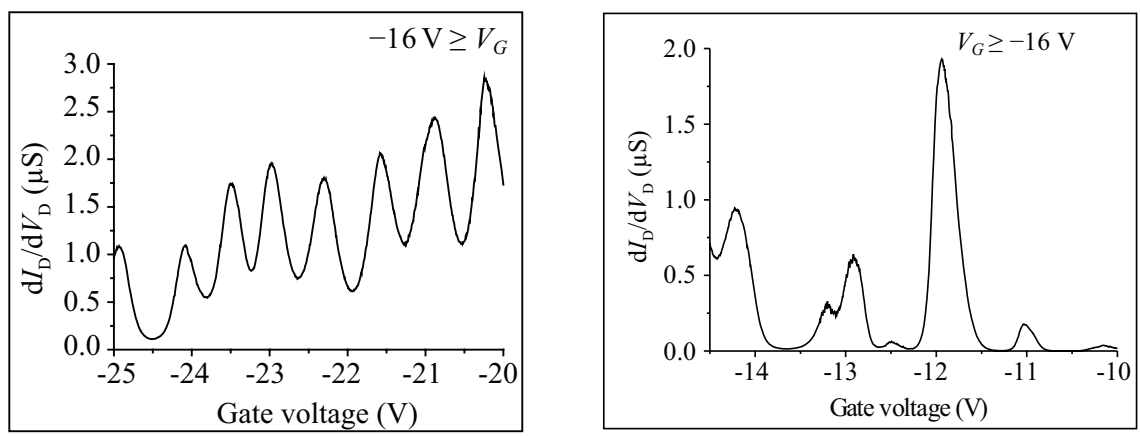

(b)

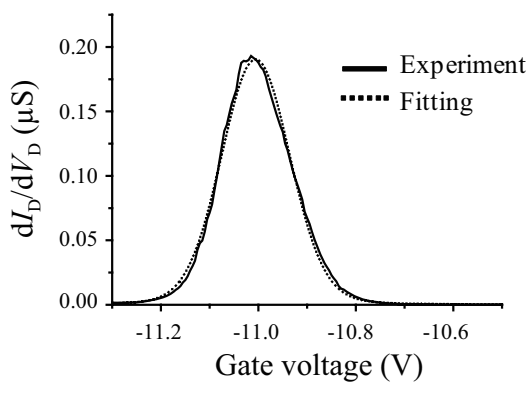

(c)

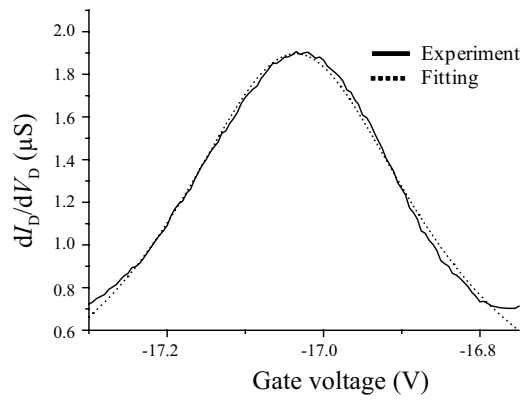

(d)

Fig. 2. (a) Differential conductance $\mathrm{d} I_{\mathrm{D}} / \mathrm{d} V_{\mathrm{D}}$ characteristic as a function of $V_{\mathrm{G}}$ at $7.3 \mathrm{~K}$. Two frequencies of oscillation were observed, which were $\Delta V_{\mathrm{G}}=1.1$ and $0.65 \mathrm{~V}$. (b) $\mathrm{d} I_{\mathrm{D}} / \mathrm{d} V_{\mathrm{D}}$ peak in linear scale at low and high $V_{\mathrm{G}}$ values. (c) $\mathrm{d} I_{\mathrm{D}} / \mathrm{d} V_{\mathrm{D}}$ peak at $V_{\mathrm{G}}=-11 \mathrm{~V}$ (black) and Gaussian fitting line (dotted). The peak is well fitted by the Gaussian function, which means that the shape of the peak can be attributed to thermal broadening. Therefore, the peak must be a Coulomb oscillation peak. (d) $\mathrm{d} I_{\mathrm{D}} / \mathrm{d} V_{\mathrm{D}}$ peak at $V_{\mathrm{G}}=-17 \mathrm{~V}$ (black) and Lorentzian fitting line (dotted). The peak is well fitted by the Lorentzian function, which means that the shape of the peak can be attributed to energy uncertainty broadening. Therefore, the peak must be a resonant tunneling current peak. 
$G=\frac{2 e^{2}}{h} \frac{4 T_{L} T_{R}}{\left(\varepsilon_{\mathrm{F}}-\varepsilon_{0}\right)^{2}+\Gamma^{2}}$, as shown in Figs. 2(c) and (d), respectively, where $e$ is the elementary charge, $\mathrm{h}$ is Planck's constant, $T_{\mathrm{L}}$ and $T_{\mathrm{R}}$ are the tunneling probabilities at the left and right tunneling barriers, $\Gamma$ is the full width at half maximum (FWHM), $\mathrm{k}$ is the Boltzmann constant, $T$ is the temperature, $\varepsilon_{\mathrm{F}}$ is the Fermi level, and $\varepsilon_{0}$ is the quantum level. The shape of the Coulomb oscillation peaks must be Gaussian, which is attributed to thermal broadening, while the resonant tunneling current peaks must be Lorentzian, which is attributed to energy uncertainty. ${ }^{(9)}$ Therefore, the $\mathrm{d} I_{\mathrm{D}} / \mathrm{d} V_{\mathrm{D}}$ oscillations at $V_{\mathrm{G}} \geq-16 \mathrm{~V}$ in Fig. 2(a) should be Coulomb oscillation peaks, and those at $V_{\mathrm{G}} \leq-16 \mathrm{~V}$ in Fig. 2(a) should be resonant tunneling current peaks. In other words, the device operates in the particle nature mode at $V_{\mathrm{G}} \geq-16 \mathrm{~V}$, and in the wave nature mode at $V_{\mathrm{G}} \leq-16 \mathrm{~V}$.

Figure 3 shows the $\mathrm{d} I_{\mathrm{D}} / \mathrm{d} V_{\mathrm{D}}$ characteristic as a function of drain voltage $V_{\mathrm{D}}$ at $7.3 \mathrm{~K}$ and $V_{\mathrm{G}}=-12.795 \mathrm{~V} . \mathrm{A} \mathrm{d} I_{\mathrm{D}} / \mathrm{d} V_{\mathrm{D}}$ peak spacing of $26 \mathrm{mV}$ is observed in the plot, which corresponds to the quantum energy level separation in the SWNT quantum island. The separation of the quantum energy levels is indicated by

$$
\Delta E_{\mathrm{Q}}=\left(\frac{h v_{\mathrm{F}}}{2 L}\right)\left[1+\left(\frac{2 L}{3 r n}\right)^{2}\right]^{-\frac{1}{2}},
$$

where $r$ is the radius of the SWNT, $L$ is the length of the SWNT, and $v_{\mathrm{F}}$ is the Fermi velocity. ${ }^{(10)}$ When $n$ increases, the equation becomes

$$
\Delta E_{\mathrm{Q}}=\frac{h v_{\mathrm{F}}}{2 L},
$$

and shows a constant value independent of $n$. The energy separation $\Delta E_{\mathrm{Q}}$ of the quantum levels for an SWNT length of $73 \mathrm{~nm}$ is calculated to be $24 \mathrm{mV}$ from eq. (2). Because this estimated value of energy separation of $24 \mathrm{mV}$ is in good agreement with the $\mathrm{d} I_{\mathrm{D}} / \mathrm{d} V_{\mathrm{D}}$

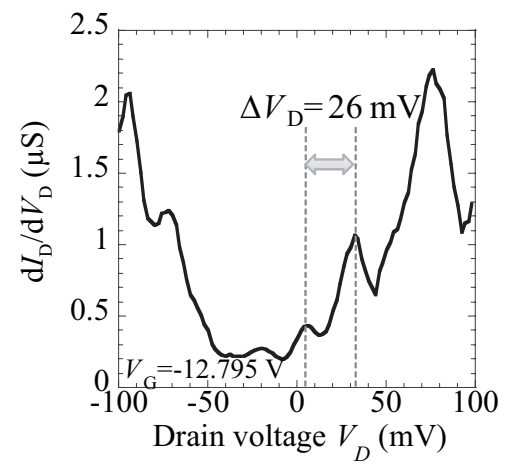

Fig. 3. $\mathrm{d} I_{\mathrm{D}} / \mathrm{d} V_{\mathrm{D}}$ characteristic as a function of drain voltage $V_{\mathrm{D}}$ at $V_{\mathrm{G}}=-12.795 \mathrm{~V}$ and $7.3 \mathrm{~K}$. The peaks are attributed to quantum energy levels in the SWNT. The estimated energy separation in 73 $\mathrm{nm} \mathrm{SWNT}$ was $24 \mathrm{meV}$, which is in good agreement with the peak spacing of $26 \mathrm{mV}$ in the plot. 
peak spacing of $26 \mathrm{mV}$ in Fig. 3, it can be concluded that the entire SWNT channel acts as a single quantum island.

Figure 4 shows a contour plot of the $\mathrm{d} I_{\mathrm{D}} / \mathrm{d} V_{\mathrm{D}}$ characteristic as a function of $V_{\mathrm{G}}$ and $V_{\mathrm{D}}$ at $7.3 \mathrm{~K}$. The characteristic can also be divided into two modes, the particle and wave

(a)
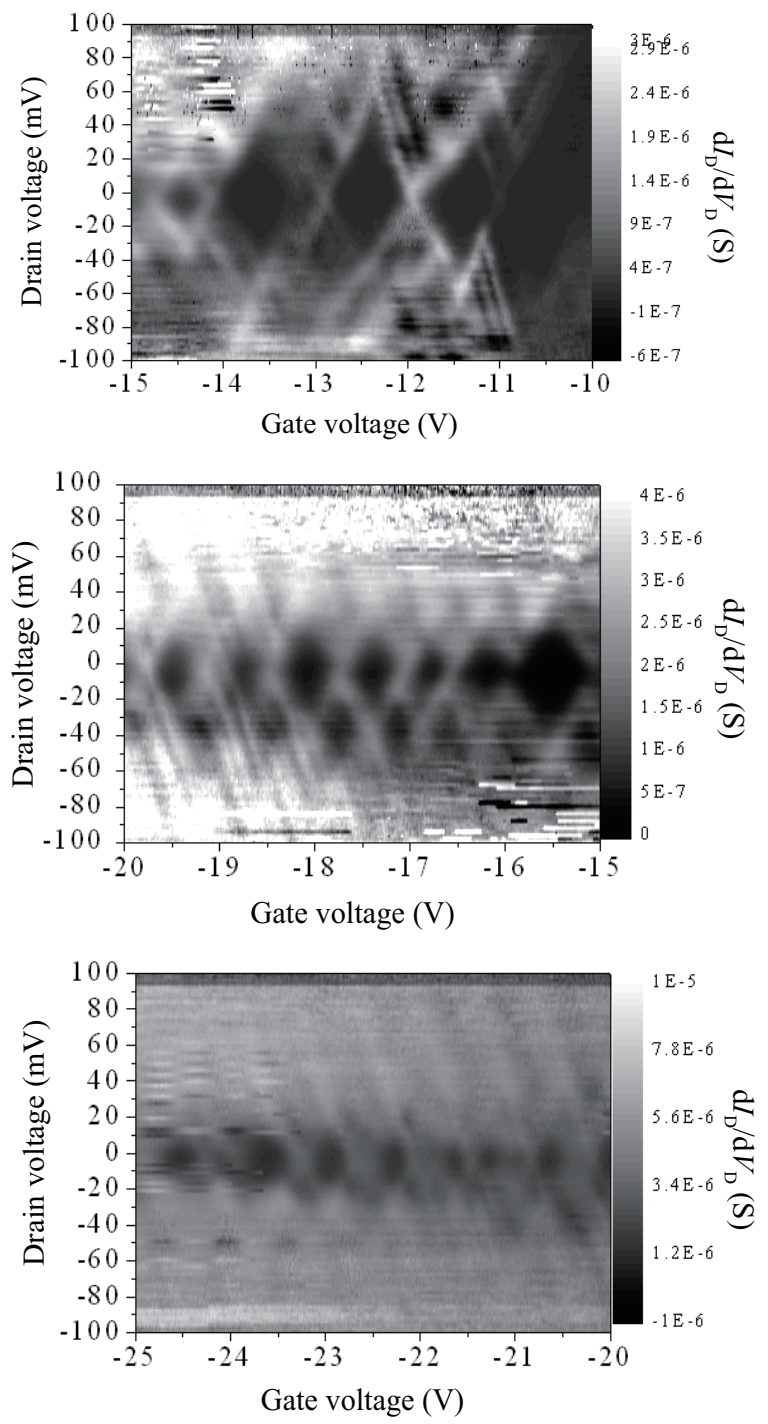

Fig. 4. Contour plot of $\mathrm{d} I_{\mathrm{D}} / \mathrm{d} V_{\mathrm{D}}$ characteristic as a function of $V_{\mathrm{G}}$ and $V_{\mathrm{D}}$ at $7.3 \mathrm{~K}$. (a) At $V_{\mathrm{G}} \geq$ $-16 \mathrm{~V}$, Coulomb diamond structures and line-shaped quantum levels outside of the Coulomb diamond structures are observed. Moreover, the Coulomb diamonds become smaller with increasingly negative $V_{\mathrm{G}}$. (b) At $-16 \mathrm{~V} \geq V_{\mathrm{G}} \geq-20 \mathrm{~V}$, the Coulomb blockade is lifted and the Coulomb diamonds disappear. The quantum levels become blurry with increasingly negative $V_{\mathrm{G}}$. The so-called Fabry-Perot quantum interference pattern is observed. (c) At $-20 \mathrm{~V} \geq V_{\mathrm{G}}$, the blurry quantum levels still remain. 
nature modes. At $V_{\mathrm{G}} \geq-16 \mathrm{~V}$, as shown in Figs. 4(a) and 4(b), the plot clearly shows the Coulomb diamond structures, which decrease in size with increasingly negative $V_{\mathrm{G}}$. Additionally, line-shaped quantum levels are observed outside of these Coulomb diamond structures. Therefore, at $V_{\mathrm{G}} \geq-16 \mathrm{~V}$, the device operated in the particle nature mode. As shown in Fig. 4(b), at $-16 \geq V_{\mathrm{G}} \geq-20 \mathrm{~V}$, the Coulomb blockade was lifted and the Coulomb diamond structures disappeared at approximately $V_{\mathrm{G}}=-16 \mathrm{~V}$. The quantum levels still remain and the so-called Fabry-Perot quantum interference pattern is observed in this region. Finally, at $V_{\mathrm{G}} \leq-20 \mathrm{~V}$, the quantum levels become blurry with increasingly negative $V_{\mathrm{G}}$, as shown in Fig. 4(c). Therefore, at $-16 \mathrm{~V} \geq V_{\mathrm{G}}$, the device operated in the wave nature mode.

Figure 5(a) shows the Coulomb charging energy $E_{\mathrm{C}}$ as a function of $V_{\mathrm{G}}$. $E_{\mathrm{C}}$ is obtained using $E_{\mathrm{C}}=\Delta E-E_{\mathrm{Q}}$, where $\Delta E$ is estimated from the size of the Coulomb diamonds. $E_{\mathrm{C}}$ markedly decreases with negatively increasing $V_{\mathrm{G}}$, almost reaching zero at $V_{\mathrm{G}} \geq-16 \mathrm{~V}$. Therefore, the Coulomb diamonds disappear at $-16 \mathrm{~V} \geq V_{\mathrm{G}}$. The tunneling capacitance $C_{\mathrm{t}}$ and the gate capacitance $C_{\mathrm{G}}$ as a function of $V_{\mathrm{G}}$ are shown in Fig. 5(b). $C_{\mathrm{t}}$ and $C_{\mathrm{G}}$ are obtained from $C_{\mathrm{t}}=e / 2 E_{\mathrm{C}}-C_{\mathrm{G}}$ and $C_{\mathrm{G}}=e / \Delta V_{\mathrm{G}}$, respectively. $C_{\mathrm{t}}$ depends on the thickness of the tunneling barrier and $C_{\mathrm{G}}$ depends on the gate structure. $C_{\mathrm{t}}$ drastically increases with negatively increasing $V_{\mathrm{G}}$, while $C_{\mathrm{G}}$ is almost constant, independent of $V_{\mathrm{G}}$. The marked increase in $C_{\mathrm{t}}$ is attributed to the decrease in the thickness of the Schottky barriers at the contacts between the SWNT quantum island and the electrodes. ${ }^{(1)}$ When the Schottky barriers become thin with negatively increasing $V_{\mathrm{G}}$, as shown in the inset of Fig. 5(b), $C_{\mathrm{t}}$ markedly increases and the coupling strength of the wave function between the outside and inside of the Schottky barrier increases. Therefore, the Coulomb blockade is lifted and $E_{\mathrm{C}}$ becomes zero at $-16 \mathrm{~V} \geq V_{\mathrm{G}}$.

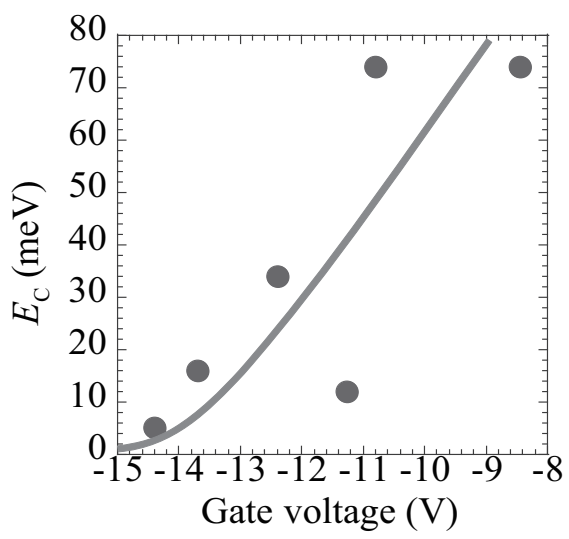

(a)

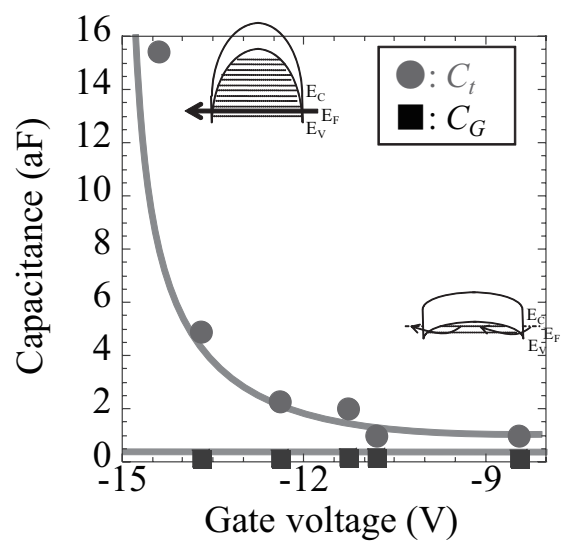

(b)

Fig. 5. (a) Coulomb charging energy $E_{\mathrm{C}}$ as a function of $V_{\mathrm{G}}$. $E_{\mathrm{C}}$ markedly decreases at $V_{\mathrm{G}} \geq$ $-16 \mathrm{~V}$ and reaches zero at approximately $V_{\mathrm{G}}=-16 \mathrm{~V}$. Therefore, the Coulomb diamonds disappear in the mid- $V_{\mathrm{G}}$ region. (b) Tunneling capacitance $C_{\mathrm{t}}$ and gate capacitance $C_{\mathrm{G}}$ characteristics as functions of $V_{\mathrm{G}}$. $C_{\mathrm{t}}$ markedly increases at $V_{\mathrm{G}} \geq-16 \mathrm{~V}$. On the other hand, $C_{\mathrm{G}}$ is almost constant, independent of $V_{\mathrm{G}}$. The marked increase in $C_{\mathrm{t}}$ is attributed to the change in the thickness of the Schottky barriers at the contact between the SWNT channel and the electrodes. 
The dependence of the FWHM of the resonant tunneling current peak characteristic on $V_{\mathrm{G}}$ is shown in Fig. 6(a). The FWHM is estimated from an $I_{\mathrm{D}}-V_{\mathrm{G}}$ plot using the Voigt function, which is a convolution of the Gaussian and Lorentzian functions, and

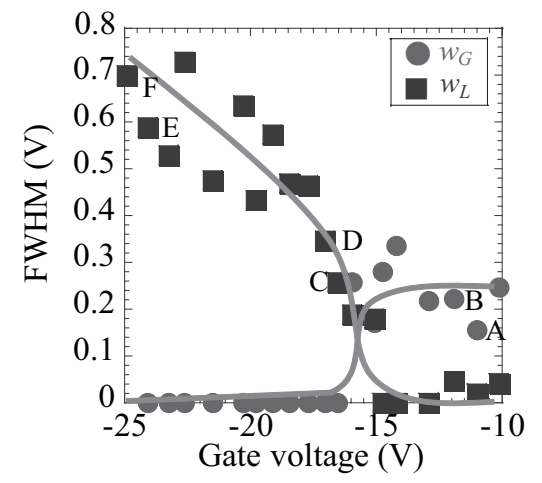

(a)

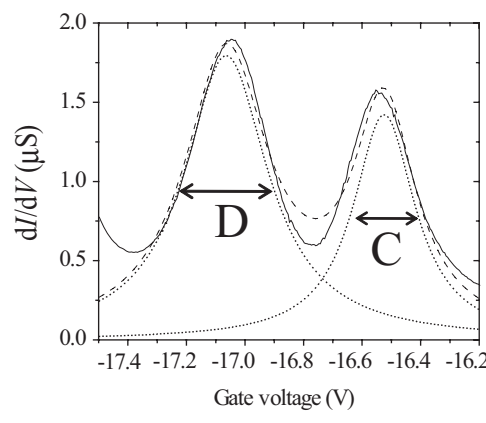

(b)

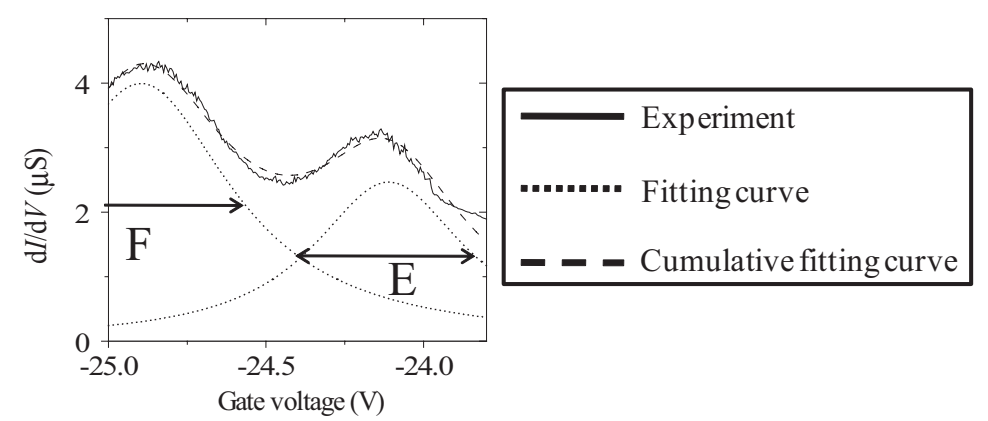

(d)

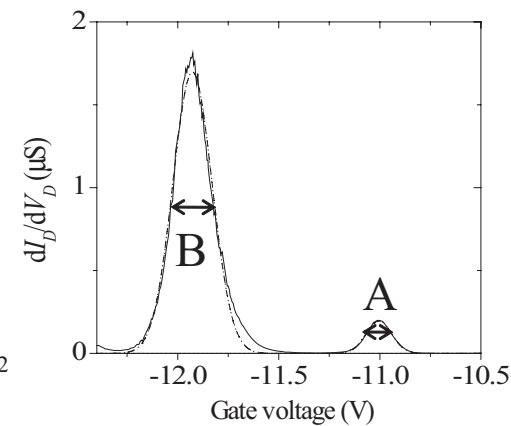

(c)
Fig. 6. (a) FWHM of the $\mathrm{d} I_{\mathrm{D}} / \mathrm{d} V_{\mathrm{D}}$ peak characteristics as a function of $V_{\mathrm{G}}$. $w_{\mathrm{G}}$ is almost constant independent of $V_{\mathrm{G}}$, and $w_{\mathrm{L}}$ is negligible at $V_{\mathrm{G}} \geq-16 \mathrm{~V}$. $w_{\mathrm{G}}$ becomes vanishingly small and $w_{\mathrm{L}}$ increases linearly with increasingly negative $V_{\mathrm{G}}$ at $-16 \mathrm{~V} \geq V_{\mathrm{G}}$. (b)-(d) FWHMs: the experimental data are indicated by the solid lines, the fitting curves are indicated by the dotted lines, the cumulative fitting curves are indicated by the dashed lines, and each alphabetic marker for a peak corresponds to a marker in the plot in Fig. 6(a). 
can be used to divide the FWHM into the FWHM of the Gaussian $w_{\mathrm{G}}$ and that of the Lorentzian $w_{\mathrm{L}}$. Figures 6(b)-6(d) show several curves fitted by the Voigt function, where the black lines are the experimental data, the dotted lines are the fitting curves, the dashed lines are the cumulative fitting curves, and each alphabetic marker for peaks A-F corresponds to a marker in the plot of Fig. 6(a). In the particle nature mode of $V_{\mathrm{G}}$ $\geq-16 \mathrm{~V}, w_{\mathrm{L}}$ is negligible and the $w_{\mathrm{G}}$ of about $0.2 \mathrm{~V}$ is independent of $V_{\mathrm{G}}$ because the FWHM of the Coulomb oscillation mainly depends on the thermal broadening of Fermi dispersion. ${ }^{(11)}$ On the other hand, in the wave nature mode of $-16 \mathrm{~V} \geq V_{\mathrm{G}}$, $w_{\mathrm{G}}$ becomes negligible and $w_{\mathrm{L}}$ increases linearly with increasingly negative $V_{\mathrm{G}} . w_{\mathrm{L}}$ is proportional to tunneling probability as follows: $\alpha w_{\mathrm{L}}=T \mathrm{~h} v_{\mathrm{F}} / 2 L$, where $\alpha$ is the ratio of modulated energy to applied $V_{\mathrm{G}}$, and $T$ is the tunneling probability. ${ }^{(12)}$ Therefore, the increase in $w_{\mathrm{L}}$ seen in Fig. 6 represents an increase in tunneling probability, which is attributed to a decrease in the thickness of the Schottky barriers with increasingly negative $V_{\mathrm{G}}$.

The logarithmic dependence of the drain current $I_{\mathrm{D}}$ on the inverse of the temperature is shown in Fig. 7. At $V_{\mathrm{G}}=-38 \mathrm{~V}$ of the wave nature mode, $I_{\mathrm{D}}$ is almost constant, which is independent of temperature. In the wave nature mode, the Schottky barrier is so thin that tunneling current becomes predominant. In contrast, at $V_{\mathrm{G}}=-10 \mathrm{~V}$ of the particle nature mode, $I_{\mathrm{D}}$ drastically increases in the high-temperature region, and the Schottky barrier height $\Delta \Phi$, as estimated from the slope, is $\Delta \Phi=50 \mathrm{mV}$. In the particle nature mode, the Schottky barriers are so thick that the thermal emission current becomes predominant in the high-temperature region. These results indicate that the tunneling probabilities at the Schottky barriers can be modulated by controlling the applied $V_{\mathrm{G}}$ and that the coupling between the SWNT quantum island and the electrodes is strong in the wave nature mode, but weak in the particle nature mode.

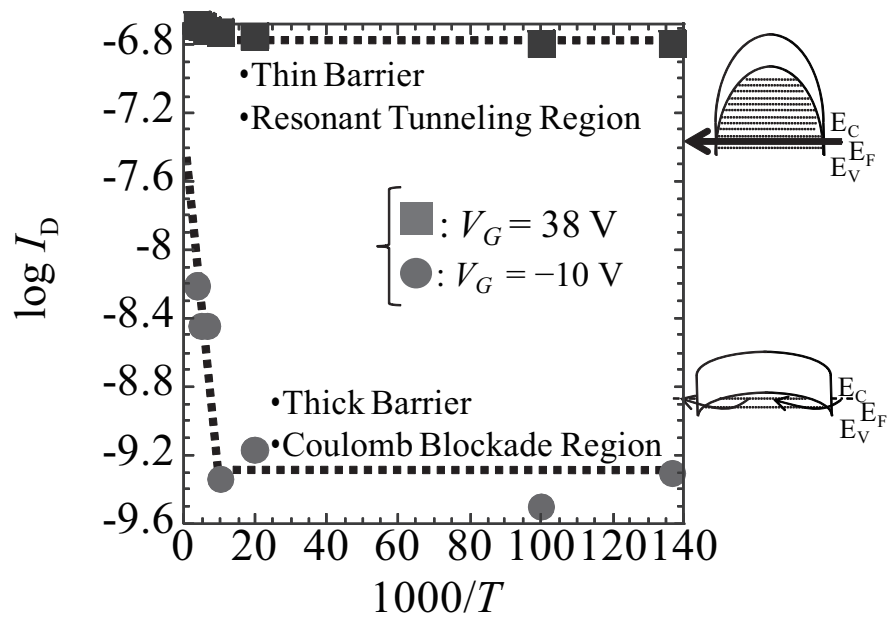

Fig. 7. Logarithmic dependence of the drain current $I_{\mathrm{D}}$ on the inverse of the temperature. At $V_{\mathrm{G}}=-38 \mathrm{~V}$ of the wave nature mode, $I_{\mathrm{D}}$ is almost constant independent of temperature. In the wave nature mode, the Schottky barrier is so thin that tunneling current becomes predominant. In contrast, at $V_{\mathrm{G}}=-10 \mathrm{~V}$ in the particle nature mode, $I_{\mathrm{D}}$ markedly increases in the high-temperature region, and the Schottky barrier height estimated from the slope is $\Delta \Phi=50 \mathrm{mV}$. 
Figure 8 shows the temperature dependence of differential conductance as a function of gate voltage. The quantum level peaks became blurry with increasing temperature. However, even at $T=100 \mathrm{~K}$, the quantum levels still remained, as indicated by arrows.

The static characteristic measurement of the device with a silicon dioxide layer on the SWNT channel shown in Fig. 9 was carried out using Agilent B 1500. In the measurement, the data were integrated for a few seconds to eliminate the effect of noise, which was set using the equipment automatically. After that, the data were recorded. From the drain current $I_{\mathrm{D}}$-gate voltage $V_{\mathrm{G}}$ characteristics of the SWNT multifunctional quantum transistor at a drain voltage of $11 \mathrm{mV}$ at $7.3 \mathrm{~K}$, the drain current showed a periodic peak-and-valley structure with two periods. As shown in Fig. 9(a), $I_{\mathrm{D}}$ was observed only in the negative- $V_{\mathrm{G}}$ region, which indicates that the measured SWNT was a p-type semiconductor. Moreover, a large period of $\sim 3 \mathrm{~V}$ at approximately $V_{\mathrm{G}}=0 \mathrm{~V}$ was observed, which was attributed to the Coulomb oscillation characteristic. Part of Fig. 9(a) is expanded in the horizontal scale and is shown in Fig. 9(b), where a small period of $\Delta V_{\mathrm{G}}=0.65 \mathrm{~V}$ was observed at a higher $V_{\mathrm{G}}$, which was attributed to the Fabry-Perot interference property ${ }^{(6)}$ of holes, the coherent length of which was more than $73 \mathrm{~nm}$, and coherent transport in the entire channel was achieved. Figure 9(c) shows a contour plot of the second-order differential conductance as a function of gate and drain voltages, in which a clear Fabry-Perot interference pattern can be seen.

From a small period of drain current oscillation of $\Delta V_{\mathrm{G}}=0.65 \mathrm{~V}$ and the equation ${ }^{(12)}$ $\Delta E_{\mathrm{Q}}=\alpha V_{\mathrm{G}}=\mathrm{h} v_{\mathrm{F}} / 2 L e$, where $\Delta E_{\mathrm{Q}}$ is the quantum energy separation, $\alpha$ is the gate modulation coefficient, $\mathrm{h}$ is Planck's constant, $v_{\mathrm{F}}$ is the Fermi velocity in graphene, $L$ is the length of the cavity of the hole, and $e$ is the elementary charge, we estimated the width of the quantum well and obtained the length of the cavity of the hole $L$ to be 55

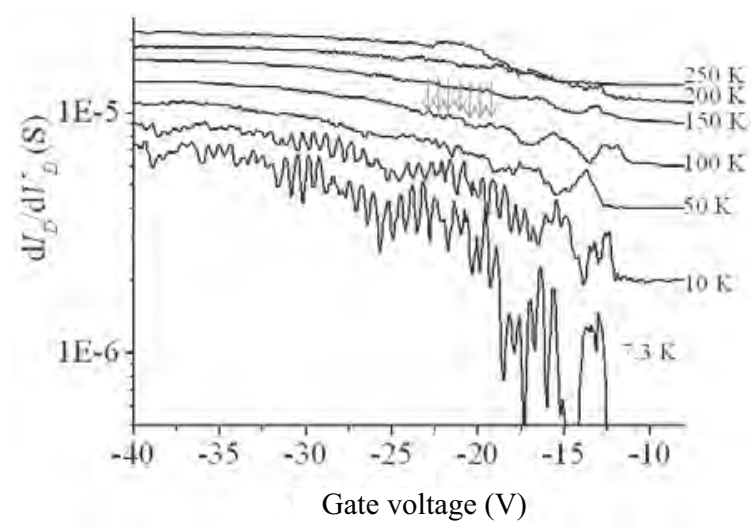

Fig. 8. Temperature dependence of differential conductance on $V_{\mathrm{G}}$. Each plot is shifted by $3 \mu \mathrm{S}$ for easier visualization. The quantum level peaks become blurry with increasing temperature. However, even at $T=100 \mathrm{~K}$, the quantum levels remain, as indicated by the arrows. 


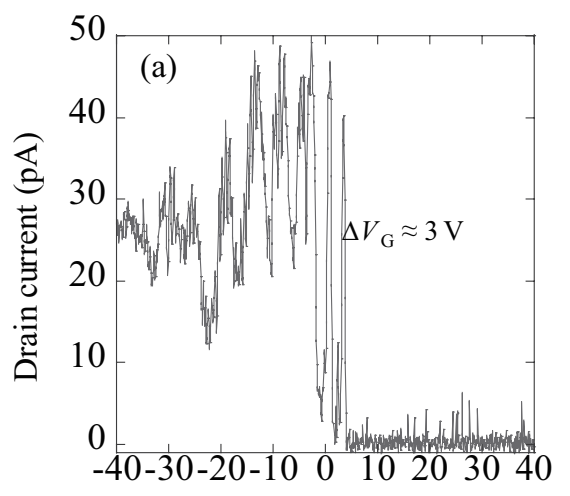

Gate voltage (V)
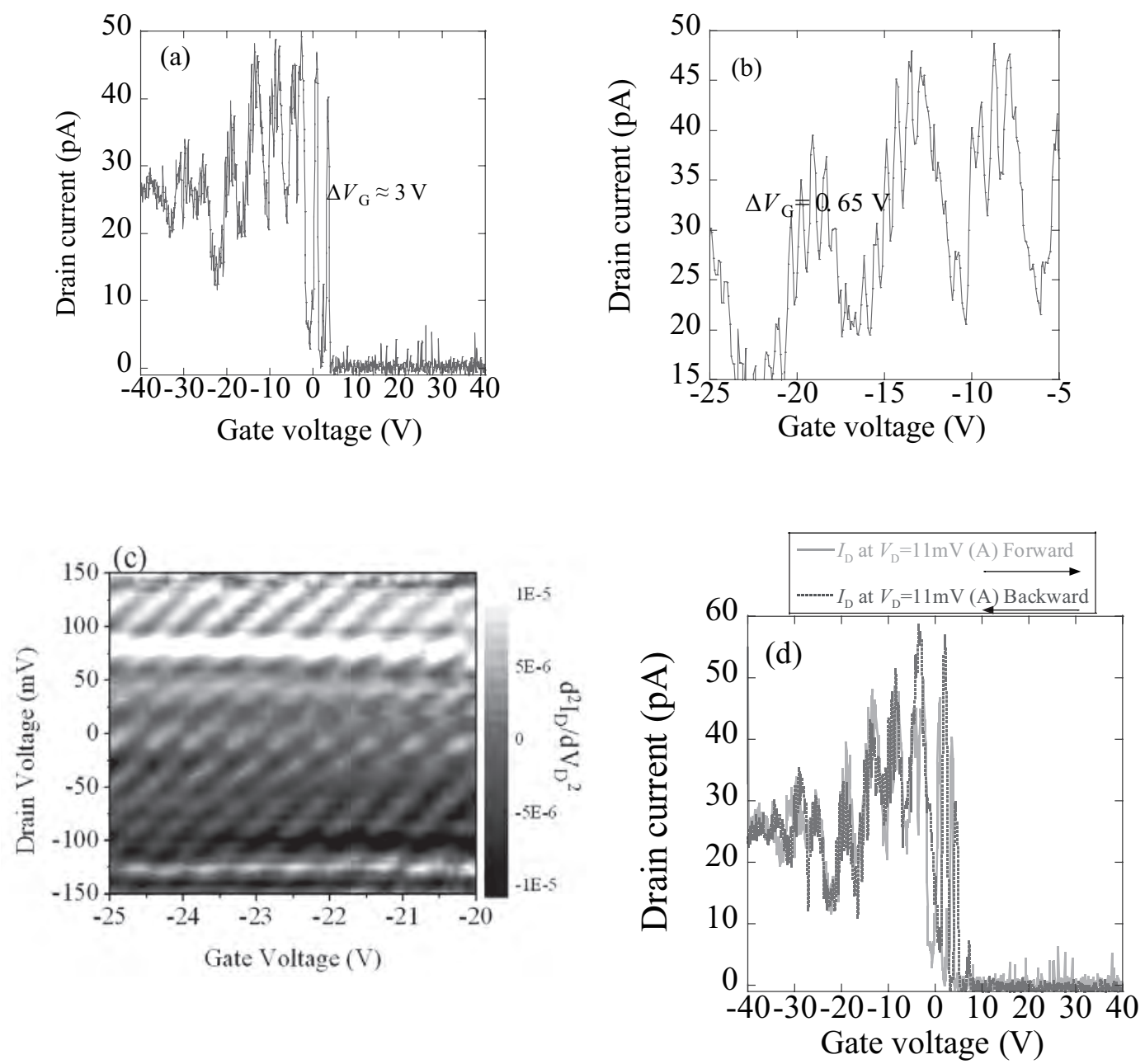

Fig. 9. (a) Coulomb oscillation characteristic at $7.3 \mathrm{~K}$. Drain current was observed only in negative-gate-voltage regions, which indicates that the measured SWNT is a p-type semiconductor. A large period of $\sim 3 \mathrm{~V}$ at an applied gate voltage of about $V_{\mathrm{G}}=0 \mathrm{~V}$ was observed, which was attributed to a Coulomb oscillation characteristic. (b) The small period of $\Delta V_{\mathrm{G}}=0.65 \mathrm{~V}$ of drain current peaks at $7.3 \mathrm{~K}$, which was attributed to the quantum interference property, namely, FabryPerot interference of a hole, the coherent length of which was more than $73 \mathrm{~nm}$. Coherent transport in the entire channel was achieved. (c) Contour plot of second-order differential conductance as a function of gate and drain voltages at $7.3 \mathrm{~K}$, which shows a clear Fabry-Perot interference pattern. (d) Drain current-gate voltage characteristics with round-trip applied gate voltage being swept from $V_{\mathrm{G}}=40$ to $-40 \mathrm{~V}$ after being swept from $V_{\mathrm{G}}=-40$ to $40 \mathrm{~V}$ at $7.3 \mathrm{~K}$. The two drain current characteristics almost overlapped. 
$\mathrm{nm}$. The estimated value is in agreement with the channel length of $73 \mathrm{~nm}$ of the device, which was obtained by scanning electron microscopy (SEM) (inset of Fig. 1). The slight difference between the estimated $L$ and the channel length must be attributed to the width of band bending in the SWNT, e.g., Schottky barriers.

In Fig. 9(d), the gray (black) line shows drain current vs applied gate voltage from $-40(40)$ to $40(-40) \mathrm{V}$ in a forward (backward) sweep of gate voltage. The SWNT multifunctional quantum transistor showed almost no hysteresis characteristics. The SWNT multifunctional quantum transistor was covered with a silicon dioxide layer, which prevents the SWNT from absorbing and releasing molecules in ambient, because the fluctuation of molecules on the SWNT usually induces the hysteresis characteristics in the drain current-gate voltage characteristics. Moreover, the SWNT multifunctional quantum transistor has a small channel length of $73 \mathrm{~nm}$, which reduces the number of trap sites near the SWNT as well as the number of trapped carriers causing hysteresis characteristics. We believe that the purification is effective for reducing the hysteresis characteristics. Thus, the hysteresis characteristic in the drain current-gate voltage characteristic was eliminated.

Figure 10(a) shows the time dependence of the conductance of the SWNT multifunctional quantum transistor at $7.3 \mathrm{~K}$ with a gate bias of $V_{\mathrm{G}}=-25.36 \mathrm{~V}$. A short sampling time of $10 \mathrm{~ms}$ was set in the dynamic characteristic measurements shown in Fig. 10. The applied gate voltage was in the Fabry-Perot interference region. The SWNT multifunctional quantum transistor shows RTS, as shown in Fig. 10(a). The RTS showed three levels, $n, n+1$, and $n+2$, of the conductance shown in Fig. 10(a). At a lower applied gate voltage, current levels higher than $n+2$ such as $n+3$ and $n+4$ appeared. The multiple levels of RTS are attributed to charge-fluctuating charge storage units near the conduction channels of the SWNT multifunctional quantum transistor. Moreover, because there was a single-charge storage unit including multiple energy levels or there were some charge storage units located at almost the same distances from the conductance channel of the SWNT multifunctional quantum transistor, the RTS appeared.

Figures 10(b) and 10(c) show histograms of the conductance levels of RTS at $V_{\mathrm{G}}=$ -25.36 and $-25.39 \mathrm{~V}$, respectively. The three peaks of the conductance levels of RTS expressed as $P_{n}, P_{n+1}$, and $P_{n+2}$ are shown in Figs. 10(b) and 10(c). The conductance levels of the three peaks directly correspond to the conductance levels of RTS. On the other hand, the relative heights of the peaks correspond to occupation probabilities at each conductance level of the RTS. The heights of the peaks depend on applied gate voltage. $P_{n}$ decreases and $P_{n+1}$ and $P_{n+2}$ increase with slightly increasing applied gate voltage from $V_{\mathrm{G}}=-25.36$ to $-25.39 \mathrm{~V}$, which means that the energy levels in the charge storage unit are modulated by applied gate voltage.

The gate voltage dependences of the natural log of the ratio between the $m_{\mathrm{th}}$ peak and the $(m+1)_{\text {th }}$ peak in the conductance histogram $P_{m+1} / P_{m}(m=n, n+1, n+2, \cdots, n+4)$ are shown in Fig. 11(a). Figures 11(b)-11(e) are enlarged plots of each $P_{m+1} / P_{m}(m=n$, $n+1, n+2, \cdots, n+4)$. The natural $\log$ of $P_{m+1} / P_{m}$ linearly depends on applied gate voltage and saturates at each $V_{\mathrm{G}}$. Each starting point of saturation is marked by an arrow in Fig. 11(a). The charge storage energy levels are floating. Therefore, the modulations of energy by $V_{\mathrm{G}}$ may differ at each charge storage unit. We think that the natural log of 

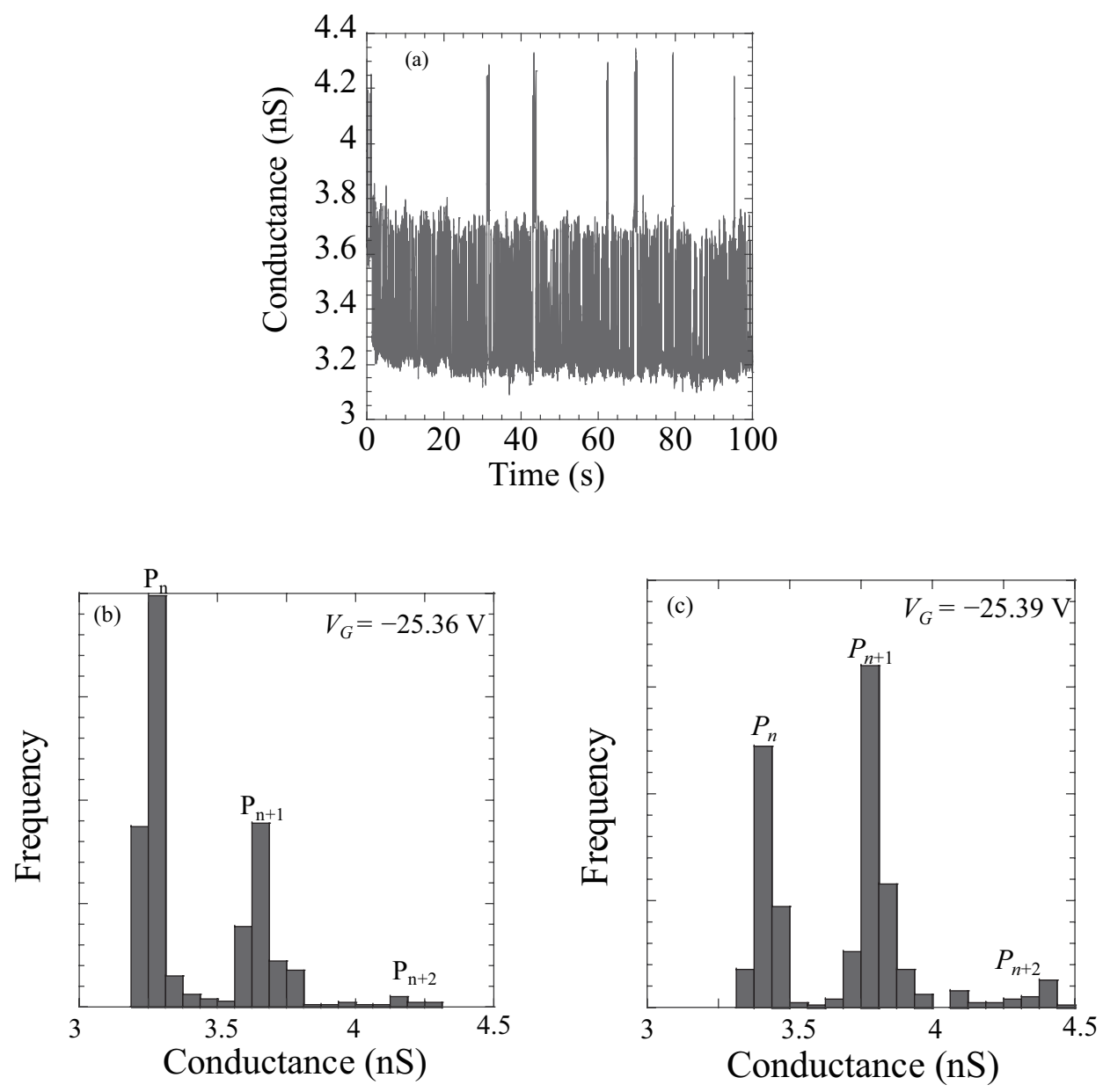

Fig. 10. (a) Time dependence of drain current with RTS at a gate voltage of $V_{\mathrm{G}}=-25.36 \mathrm{~V}$. The time dependence of drain current was sampled for $100 \mathrm{~s}$ and the sampling time was $10 \mathrm{~ms}$; (b) and (c) show histograms of the conductance levels of RTS at $V_{\mathrm{G}}=-25.36$ and $-25.39 \mathrm{~V}$, respectively. $P_{n}$ decreases and $P_{n+1}$ and $P_{n+2}$ increase with slightly increasing applied gate voltage from $V_{\mathrm{G}}=-25.36$ to $-25.39 \mathrm{~V}$.

$\mathrm{P}_{m+1} / \mathrm{P}_{m}$ saturates at each $V_{\mathrm{G}}$ owing to the difference in energy modulation by $V_{\mathrm{G}}$ at each charge storage unit.

The charge transition is modeled, as shown in Fig. 12(a), in which the energy barrier is between the SWNT and the charge storage unit. The energy difference $\Delta E_{n}$ between the charge storage energy level $E_{n}$ and the Fermi level $E_{\mathrm{f}}$ is expressed as $\Delta E_{n}=E_{\mathrm{f}}-E_{n}$, which is modulated by the applied gate voltage $V_{\mathrm{G}}$. According to equilibrium statistical mechanics, $P_{m+1} / P_{m}$ is given by ${ }^{(13)}$ 

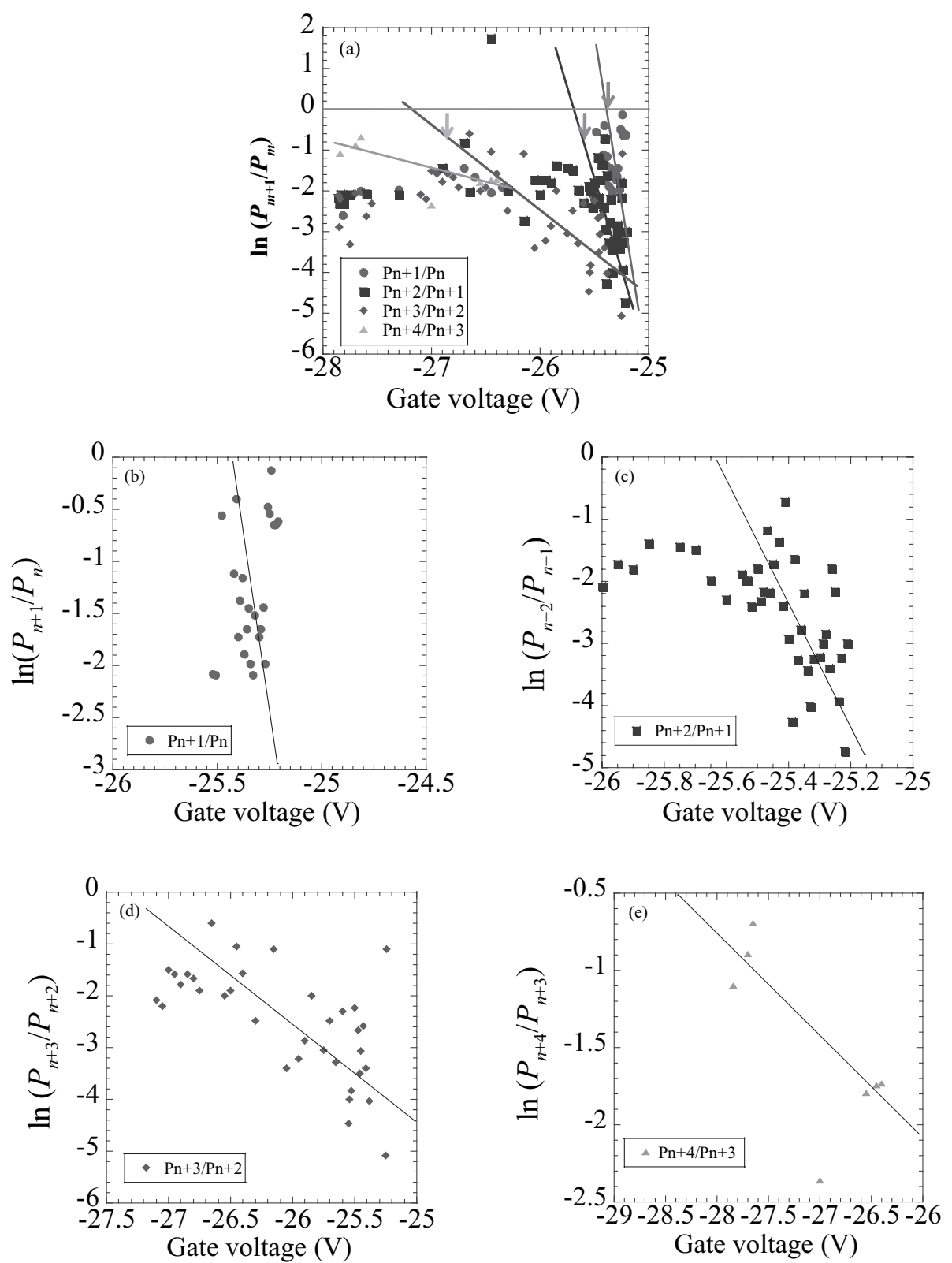

Fig. 11. (a) Gate voltage dependence of the natural log of the ratio between the occupancy probabilities of the $m_{\text {th }}$ current levels $P_{n+1} / P_{n}$. (b)-(e) Enlarged plots of each $P_{m+1} / P_{m}(m=n, n+1$, $n+2, \cdots n+4)$. The natural $\log$ of $P_{n+1} 1 / P_{n}$ was linearly dependent on gate voltage, the slopes of which are $-10.4,-4.78,-1.47$, and $-0.690 \mathrm{~V}^{-1}$, respectively. 


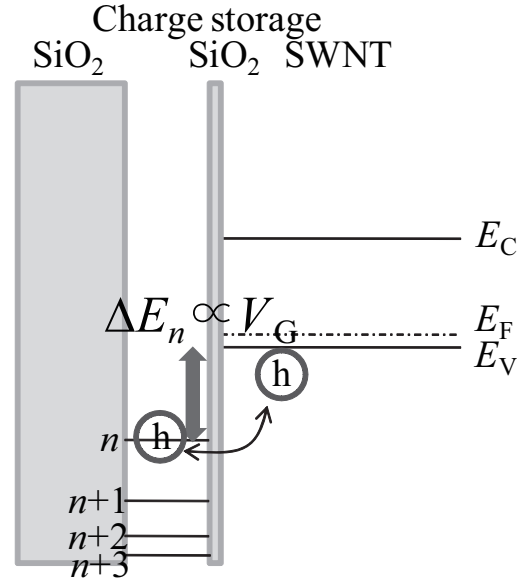

(a)

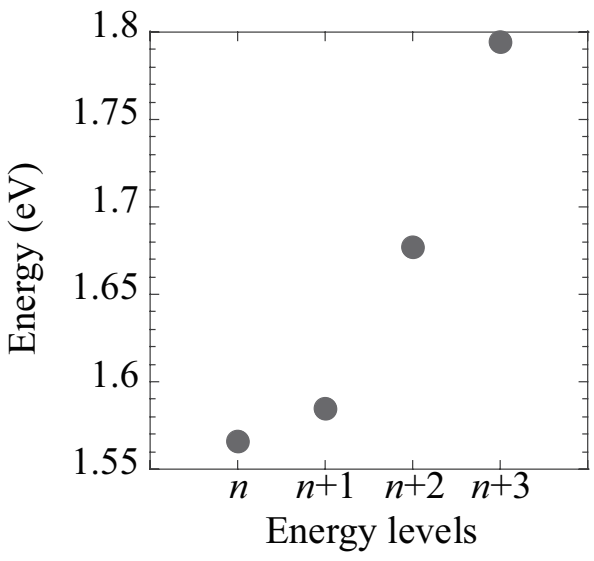

(b)

Fig. 12. (a) Schematic model of charge storage. When the charge storage energy level is coincident with the top of the valence band owing to applied gate voltage, the carrier departs and arrives between them through the barrier with tunneling. The exiting probabilities of the carrier at the charge storage and valence band depend on the relative height of their energy levels under equilibrium condition. (b) Estimated charge storage energy levels from the dependence of $P_{n+1} / P_{n}$ on $V_{\mathrm{G}}$ shown in Figs. 11(b)-11(e) and eq. (3). The energy levels increase from 0.17 to $0.28 \mathrm{eV}$ with increasing number of energy levels in the region of $V_{\mathrm{G}}$ from -25 to $-28 \mathrm{~V}$.

$$
P_{m+1} / P_{m}=\left(g_{\mathrm{f}} / g_{\mathrm{S}}\right) e^{-\beta\left(E_{\mathrm{f}}-E_{n}\right)}=\left(g_{\mathrm{f}} / g_{\mathrm{S}}\right) e^{-\beta \Delta E_{n}},
$$

where $g_{\mathrm{f}}$ and $g_{\mathrm{S}}$ are the degeneracies of the top of the valence band and the charge storage unit, respectively. $g_{\mathrm{f}} / g_{\mathrm{S}}$ is assumed to be 1 . $\beta$ is $1 / \mathrm{k} T$. $E_{n}$ includes the contributions of the $e$ electrostatic potential induced by $V_{\mathrm{G}}$, the intrinsic energy level in the storage unit, and the Coulomb charging energy. The basis of eq. (3) is the Arrhenius equation. In this model, the height of the barrier is the energy difference between $E_{\mathrm{f}}$ and $E_{n}$. Assuming a linear dependence of $\Delta E_{n}$ on $V_{\mathrm{G}}, \Delta E_{n}$ can be written as $\Delta E_{n}=\alpha e\left(V_{0}-V_{\mathrm{G}}\right)$, where $\alpha$ is the gate modulation coefficient that has a constant value of 0.062. $\alpha$ is obtained from the periods of Fabry-Perot interference characteristic on $V_{\mathrm{D}}$ and $V_{\mathrm{G}} . V_{0}$ is the offset voltage obtained from the intersecting points of the extrapolating line of the fitting lines and the line of $\ln \left(P_{m+1} / P_{m}\right)=0$. Therefore, eq. (3) is transformed to

$$
\ln \left(P_{m+1} / P_{m}\right)=-\beta e \alpha\left(V_{0}-V_{\mathrm{G}}\right) .
$$

Equation (4) is the transformed Arrhenius equation, in which $V_{\mathrm{G}}$ is the parameter.

From the dependence of $P_{n+1} / P_{n}$ on $V_{\mathrm{G}}$ shown in Fig. 11 and eqs. (3) and (4), $\Delta E_{n}$ can be obtained and is shown in Fig. 12(b). The obtained energy levels are from 1.57 to $1.79 \mathrm{eV}$. 


\section{Summary}

In summary, we succeeded in fabricating and demonstrating a multifunctional quantum transistor using the particle and wave natures of holes in SWNT. This transistor can operate in the wave nature mode as an RTT and in the particle nature mode as an SHT. We were able to reveal that the principle of the characteristic transition from an SHT to an RTT is the modulation of the coupling strength between the SWNT quantum island and the electrodes by the applied $V_{\mathrm{G}}$.

The fabrication of the charge storage unit, the detection of single-charge transitions near the channel of the SWNT multifunctional quantum transistor at $7.3 \mathrm{~K}$, and the estimation of the energy differences between the energy levels of the SWNT multifunctional quantum transistor and the storage carriers have been demonstrated. The observed RTS was dependent on applied $V_{\mathrm{G}}$. We were able to estimate the energy levels in the charge storage unit from the gate voltage dependence of RTS.

\section{References}

1 A. Javey, J. Guo, Q. Wang, M. Lundstrom and H. Dai: Nature 424 (2003) 654.

2 S. Heinze, J. Tersoff, R. Martel, V. Derycke, J. Appenzeller and Ph. Avouris: Phys. Rev. Lett. 89 (2002) 106801-1.

3 R. Martel, V. Derycke, C. Lavoie, J. Appenzeller, K. K. Chan, J. Tersoff and Ph. Avouris: Phys. Rev. Lett. 87 (2001) 256805-1.

4 T. Kamimura and K. Matsumoto: Jpn. J. Appl. Phys. 44 (2005)1603.

5 Y. Nosho, Y. Ohno, S. Kishimoto and T. Mizutani: Nanotechnology 17 (2006) 3412.

6 W. Liang, M. Bockrath, D. Bozovic, J. H. Hafner, M. Tinkham and H. Park: Nature 411 (2001) 665 .

7 M. Suzuki, K. Ishibashi, T. Ida, D. Tsuya, K. Toratani and Y. Aoyagi: J. Vac. Sci. Technol. B 19 (2001) 2770.

8 W. Kim, A. Javey, O. Vermesh, Q. Wang, Y. Li, and H. Dai: Nano Lett. 3 (2003) 193.

9 M. Radosavljevic', M. Freitag, K. V. Thadani, and A. T. Johnson: Nano Lett. 2 (2002) 761.

10 T. Kamimura and K. Matsumoto: IEICE Trans. Electron. E87-C (2004) 1795.

11 E. B. Foxman, P. L. McEuen, U. Meirav, N. S. Wingreen, Y. Meir, P. A. Belk and S. J. Wind: Phys. Rev. B 47 (1993) 10020.

12 T. Kamimura and K. Matsumoto: Jpn. J. Appl. Phys. 45 (2006) 338.

13 H. B. Peng, M. E. Hughes and J. A. Golovchenkoa: Appl. Phys. Lett. 89 (2006) 243502. 